OPEN ACCESS

Edited by:

Yateendra Mishra,

Queensland University of Technology,

Australia

Reviewed by:

Bo Yang,

Kunming University of Science and

Technology, China

Xiasheng Shi,

China University of Mining and

Technology, China

${ }^{*}$ Correspondence:

Waqas Ahmed

waqas@pieas.edu.pk

Keum-Shik Hong

kshong@pusan.ac.kr

Specialty section: This article was submitted to

Smart Grids,

a section of the journal Frontiers in Energy Research

Received: 08 June 2021 Accepted: 20 January 2022 Published: 28 February 2022

Citation:

Alvi U-E-H, Ahmed W, Hong K-S,

Rehan M and Ahmed S (2022)

Optimality Condition and Distributed Optimization for Economic Dispatch Using a Novel Weighted Incremental

Cost Consensus Approach.

Front. Energy Res. 10:722256.

doi: 10.3389/fenrg.2022.722256

\section{Optimality Condition and Distributed Optimization for Economic Dispatch Using a Novel Weighted Incremental Cost Consensus Approach}

\author{
Um-E-Habiba Alvi ${ }^{1}$, Waqas Ahmed ${ }^{1 *}$, Keum-Shik Hong ${ }^{2 *}$, Muhammad Rehan ${ }^{1}$ and \\ Shakeel Ahmed ${ }^{1}$
}

${ }^{1}$ Department Electrical Engineering, Pakistan Institute of Engineering and Applied Sciences (PIEAS), Islamabad, Pakistan, ${ }^{2}$ School of Mechanical Engineering, Pusan National University, Busan, South Korea

This research report investigates a novel optimization approach for the economic dispatch problem (EDP) based on the weighted sum of generators' costs under supply-demand balance. Unlike conventional approaches, we present a distributed optimization approach that ensures optimality using weighted incremental cost (IC) consensus and signconsensus error convergence. We can apply the optimization of a weighted sum of generators' costs to address several constraints, such as capacity and environmental constraints, in addition to the supply-demand balance. The proposed distributed weighted incremental cost consensus approach has been applied to the IEEE-30 bus and IEEE-118 bus systems over a communication topology. The results indicate the efficacy of weights to address generation constraints and the convergence of weighted ICs under supplydemand balance.

Keywords: distributed optimization, economic dispatch, consensus protocol, weighted incremental cost, IEEE 30bus system

\section{INTRODUCTION}

Technological progress and changes in ecological policies around the world are forcing the energy markets to follow distributed generation models (El-Baz et al., 2019; Flore et al., 2019) and (Schubert and Stadelmann, 2015). To this end, economic dispatch becomes a fundamental problem [see and (Guozden et al., 2020; Mao et al., 2019)] in which total power generation cost of all distributed generating units is minimized over a micro-grid (Chansareewittaya, 2018). Most of the existing economic dispatch problem (EDP) algorithms use a centralized approach (Krishnamurthy et al., 2017; McLarty et al., 2019), which however cannot be applied to distributed generation scenarios (Liu et al., 2016; Zhang et al., 2019; Wang et al., 2020). Recently, a lot of work has been focused on solving EDP problem in a distributed scenario. For example, the study in Guodong Liu et al. (2019) developed a distributed energy management system for a public micro-grid to schedule distributed energy resources and energy storage systems, based on price signals. Yun et al. (2019) resolved the EDP in a distributed manner through a simple algorithm that required no particular initialization. The work in Yi et al. (2020) proposed a distributed method for the economic multi-energy system by considering numerous equality and inequality constraints for recurrent renewable generations. The authors in Lü et al. (2020) developed a directed and distributed Lagrangian momentum algorithm that joined the gradient-tracking technique with momentum terms by using nonuniform step-sizes. It has been observed that machine learning and optimization methods have several engineering and 
biomedical applications, as observed in the recent studies (Rehan and Hong, 2011; Ansari et al., 2019; Wu et al., 2019; Hou et al., 2020; Zafar and Hong, 2020; Zhu et al., 2021). Such bioinspired and learning schemes have their applications in artificial intelligence and adaptive systems (Rehan et al., 2011; Iqbal et al., 2018; Gomez-Tames et al., 2019; Ma et al., 2019; Manzanera et al., 2019; Yang et al., 2019; Chiarelli et al., 2020; Liu et al., 2020; Wei et al., 2020; Dalin Yang et al., 2020).

Recently, consensus algorithms have been applied to attain a distributed EDP (Yun et al., 2019; Wang and Liu, 2020), which are simple to realize and guarantee an optimal solution by respecting various communication topologies in a micro-grid. A robust consensus-based approach has been investigated in Zhang et al. (2016) for studying the potentials of distributed methods over environmental constraints, ramp-rate limit, prohibited zones, and switching topologies, in addition to box and equality constraints. An advanced concept of learning and adaptation has been employed in Zhang et al. (2018) via a two-layer and leader-based approach. In Chen et al. (2019), the authors considered an economical distributed control approach for the battery energy storage system under frequency control, and an incremental cost (IC) consensus algorithm was developed. Wang and Liu (2020) represented two distributed IC consensus-based optimization algorithms to answer EDPs without and with generation constraints via small gain theorem. Chen et al. (2020) presented a distributed consensus approach for cost-effective load dispatch under frequency regulation. The works in Wang et al. (2019), Yu et al. (2020), and Xing et al. (2019) proposed EDP methods under random delay, for time-varying step-sizes, and transmission losses. The role of control theories and Lyapunov analysis (us Saqib et al., 2018; Tang and Li, 2019; Zhao et al., 2019; Gao and Liu, 2020; Santos et al., 2020; Yao et al., 2020) can be useful in attaining optimization issues. The stability analysis, as observed in Dev and Sarkar (2019), Perng et al. (2020), Souza et al. (2020), Xie et al. (2020), and Yin et al. (2020), can be applied to attain coordination in generation systems.

The above-mentioned central, distributed, and IC consensusbased distributed methods consider optimization of total cost of generation, which is the sum of costs of all generating units. It should be noted that rather than achieving optimal generation, optimization of weighted sum of generations is more meaningful, as it can be applied to comply with several constraints, such as capacity constraints, environmental restraints, and other auxiliary restrictions. The idea of inclusion of weights in optimization is interesting, as it can provide freedom to designers for appropriately minimizing the total generation cost and to define and apply user-defined constraints as per requirements of the generation system. However, the solution to this problem is equally challenging for a distributed EDP environment, as the conventional optimality conditions and the conventional consensus methods cannot be applied in this situation. Motivated by these concerns, the present work is a step toward formulating and establishing conditions for distributed EDP with weighted cost function under supply-demand constraints, which can consider various generation constraints as well. Specifically, the contributions in the paper are as follows.
- A new problem of distributed optimization in EDP has been formulated to optimize the weighted costs of generators, for which the conventional EDP can be written as a specific case. To the best of our knowledge, a distributed optimization approach for solving weighted EDP (Eq. 3) has been provided for the first time. The proposed optimization approach is also advantageous, as it can be applied to attain several constraints in a simple manner by adjusting weights.

- Two optimality conditions for the said problem are investigated: The first condition provides the generic optimality condition, based on Lagrangian methods, and it shows that the optimal weighted EDP can be resolved by addressing weighted IC consensus, rather than the conventional IC consensus. The second condition investigates the optimality condition from a distributed optimization point of view over a network, based on sign-consensus error convergence, rather than applying the conventional consensus error.

- A novel protocol for the distributed weighted EDP has been provided. The condition on the protocol parameters for attaining EDP's optimal solution is provided by applying graph theory and Lyapunov analysis. The proposed weighted EDP has been applied to the IEEE-30 bus and IEEE-118 bus systems, and the comparison of the proposed method with the existing ones is studied.

- It is worth mentioning that the proposed approach supports the signed graphs for the weighted cost optimization. The signed graphs can arise between two generators due to transmission of information via amplification through inverting amplifiers.

- By the application of the recent results in Pourbabak et al. (2020) for optimal power flow (OPF), a modified weighted cost function-based approach is also provided to resolve a more advanced and complicated problem, considering the practical constraints in addition to the cost optimization.

\section{PROBLEM FORMULATION}

The neighborhood connections between generators can be modeled by means of a bidirectional graph $\mathcal{G}=(\mathcal{V}, \mathcal{E}, \mathcal{A})$, where $\mathcal{V}, \mathcal{E}$, and $\mathcal{A}$ represent sets of nodes, edges, and adjacency matrix, respectively. The entries of $\mathcal{A}$ are represented by $a_{i j}$ to depict communication links between generators. Consider the following equation (Krishnamurthy et al., 2017):

$$
C_{i}\left(P_{G_{i}}\right)=\alpha_{i}+\beta_{i} P_{G_{i}}(t)+\gamma_{i} P_{G_{i}}^{2}(t),
$$

which represents the cost expression of $i$ th power generator with $\alpha_{i}, \beta_{i}$ and $\gamma_{i}$ as coefficients. The weighted total cost can be written as

$$
C_{w t}\left(P_{G_{i}}\right)=\sum_{i=1}^{N} w_{i} C_{i}\left(P_{G_{i}}\right),
$$

where $w_{i}$ for $i=1,2, \ldots, N$ are positive weights and $C_{w t}$ shows the weighted cost of generation. In relation (2), we have considered a 
modified cost function with weights for attaining the constraints. The use of weights is interesting and has found applications in the literature. For instance, weights have been considered in the cost functions (Shadmand et al., 2019) for attaining multiple control objectives for power electronic interfaces. Co-variance weighted nonlinear least-square cost functions are presented in Pintelon et al. (1997) for model identification applications. Time-delay estimation over highly oscillatory objective functions, with applications in sonar, as observed in $\mathrm{Wu}$ and $\mathrm{Li}$ (1998), has been attained for Cramer-Rao bounds [see also Toh and Eng (2008) for weighted least-square learning]. Even weights have also been used in economic dispatch problem over multiple objective functions of cost and environment (Dong and Wang, 2020), which motivates us to use weights for attaining EDP constraints.

Remark 1. The total generation cost can be written as $C_{t}\left(P_{G_{i}}\right)=\sum_{i=1}^{N} C_{i}\left(P_{G_{i}}\right)$. Note that the minimization of $C_{w t}\left(P_{G_{i}}\right)$ can ensure reduction of $C_{t}\left(P_{G_{i}}\right)$. If $w_{i}=1$, we have $C_{w t}\left(P_{G_{i}}\right)=C_{t}\left(P_{G_{i}}\right)$, that is, the weighted cost function reduces to the conventional (nonweighted) cost function. The weights can be selected such that $\frac{1}{N} \sum_{i=1}^{N} w_{i}=1$. Here, the advantage is that these weights can be selected for attaining user-defined requirements. For instance, if a generator is likely to reach its upper generation constraint, its weight can be adjusted to a higher value to give more weight contribution to the minimization of the generator's cost function. If a generator (say generator 10) is environmental friendly, its weight $w_{10}$ can be taken smaller for less weight on the optimization of $C_{10}\left(P_{G_{10}}\right)$. Hence, power generation $P_{G_{i}}$ of a generator $i$ can be reduced by increasing its weight $w_{i}$, and vice versa.

Consider the problem of minimization of weighted cost of generators under supply-demand constraint for generator capacity constraint, given by

$$
\left\{\begin{array}{c}
\min C_{w t}\left(P_{G_{i}}\right)=\sum_{i=1}^{N} w_{i} C_{i}\left(P_{G_{i}}\right), \\
\text { subjectto } P_{D}=\sum_{i=1}^{N} P_{G_{i}}, \\
P_{G_{i}} \in\left[\begin{array}{ll}
P_{G_{i}}^{m} & P_{G_{i}}^{M}
\end{array}\right] .
\end{array}\right.
$$

The relation (Eq. 3) represents the proposed optimization problem, aiming to minimize the weighted cost $\left(C_{w t}\right)$ in such a way that the power demand remains equal to summation of generated power by all generators. Condition (Eq. 4) shows that generated power should belong to maximum $\left(P_{G_{i}}^{M}\right)$ and minimum $\left(P_{G_{i}}^{m}\right)$ power of generator, called capacity constraint. In the present work, we have considered the minimization of weighted cost function $C_{w t}\left(P_{G_{i}}\right)$ under the supply demand equality constraint $P_{D}=\sum_{i=1}^{N} P_{G_{i}}$. The box constraint of (Eq. 4) is not explicitly considered in the work. Rather, this constraint is indirectly ensured via selection of weights $w_{i}$ and $w_{j}$. Generally, the power generation limits can be determined by the minimum and maximum capacity of a generator, based on the design, technology used, and type and amount of fuel applicable for a distributed generator. These details are provided by the manufacturers/vendors. It should be noted that the weights $w_{i}$ cannot be unified in the objective function of (Eq. 3), because the box constraint in (Eq. 3) cannot be considered a weighted supplydemand constraint. The optimization problem (Eq. 3) becomes complex owing to different formats of objective function (weighted) and constraints (nonweighted) and due to consideration of distributed as well as dynamic optimization over a graph. Similar to $\mathrm{He}$ et al. (2019), the total power generation mismatch $(\Delta P)$ is given by

$$
\Delta P=P_{D}-\sum_{i=1}^{N} P_{G_{i}} .
$$

If we take the derivative of Eq. 1 with respect to $P_{G_{i}}$, we obtain the IC of $i$ th unit as

$$
\eta_{i}(t)=\frac{d C_{i}\left(P_{G_{i}}\right)}{d P_{G_{i}}}=\beta_{i}+2 \gamma_{i} P_{G_{i}} .
$$

\section{MAIN RESULTS}

\section{Investigation of Optimality Condition}

This subsection investigates the optimality conditions for the proposed EDP problem in Eq. 3.

Lemma 1. The optimal solution $P_{G_{i}}^{*}$ for the weighted EDP in (Eq. 3) can be attained if

$$
\begin{gathered}
w_{i} \frac{d C_{i}\left(P_{G_{i}}\right)}{d P_{G_{i}}}=w_{j} \frac{d C_{j}\left(P_{G_{j}}\right)}{d P_{G_{j}}}, \forall i, j=1,2, \ldots, N, \\
P_{D}=\sum_{i=1}^{N} P_{G_{i}} .
\end{gathered}
$$

Proof. Consider the Lagrangian function as

$$
L\left(P_{G_{i}}, \lambda\right)=\sum_{i=1}^{N} w_{i} C_{i}\left(P_{G_{i}}\right)+\lambda\left(P_{D}-\sum_{i=1}^{N} P_{G_{i}}\right) .
$$

Taking the derivative of $L\left(P_{G_{i}}, \lambda\right)$ with respect to $P_{G_{i}}$ and $\lambda$, we obtain

$$
\begin{gathered}
\frac{\partial L\left(P_{G_{i}}, \lambda\right)}{\partial P_{G_{i}}}=w_{i} \frac{\partial C_{i}\left(P_{G_{i}}\right)}{\partial P_{G_{i}}}-\lambda=0 . \\
\frac{\partial L\left(P_{G_{i}}, \lambda\right)}{\partial \lambda}=P_{D}-\sum_{i=1}^{N} P_{G_{i}}=0 .
\end{gathered}
$$

For optimality condition, we require $\frac{\partial L\left(P_{G_{i}}, \lambda\right)}{\partial P_{G_{i}}}=0$ and $\frac{\partial L\left(P_{G_{i}}, \lambda\right)}{\partial \lambda}=0$, which lead from (Eq. 10)-(Eq. 11) that

$$
\begin{gathered}
w_{i} \frac{\partial C_{i}\left(P_{G_{i}}\right)}{\partial P_{G_{i}}}=\lambda . \\
P_{D}-\sum_{i=1}^{N} P_{G_{i}}=0 .
\end{gathered}
$$

The conditions in Eqs 12 and 13 are equivalent to (Eqs 7, 8), respectively, which completes the proof. 
Remark 2. Lemma 1 applies the Lagrangian function approach to provide a generic optimality condition for (Eq. 3). It states that the optimization of weighted $\operatorname{EDP}$ (Eq. 3) can be attained by attaining weighted IC consensus (Eq. 7) and supply-demand balance condition (Eq. 8). For $w_{i}=w_{j}=1$ with $i, j=1,2, \ldots, N$, the conventional IC consensus scheme as in Chen et al. (2019) and Yu et al. (2020) can be derived a specific result.

Now, we derive an optimality condition from distributed optimization over a network point of view. Let us define the weighted IC consensus error as

$$
e_{i}(t)=w_{i} \eta_{i}(t)-\frac{1}{N} \sum_{j=1}^{N} w_{j} \eta_{j}(t) .
$$

Lemma 2. The optimal solution $P_{G_{i}}^{*}$ for the weighted EDP (Eq. 3) can be attained if $e_{i}(t)=w_{i} \eta_{i}(t)-\frac{1}{N} \sum_{j=1}^{N} w_{j} \eta_{j}(t)$ converges to the zero and $\sum_{i=1}^{N} P_{G_{i}}$ reaches at $P_{D}$.

Proof. Let us define $e(t)=\left[e_{1}^{T}, e_{2}^{T}, \ldots, e_{N}^{T}\right]^{T}$, $\eta(t)=\left[\eta_{1}^{T}, \eta_{2}^{T}, \ldots, \eta_{N}^{T}\right], \quad 1_{N}=[1,1, \ldots, 1]^{T} \in R^{N}$, and $W=\operatorname{diag}\left\{w_{1}, w_{2}, \ldots, w_{N}\right\}$, the condition (Eq. 14) gives

$$
e(t)=\left(I-\frac{1}{N} 1_{N} 1_{N}^{T}\right) W \eta(t) .
$$

It can be observed that zero is a simple eigenvalue of $\left(I-\frac{1}{N} 1_{N} 1_{N}^{T}\right)$, corresponding to right eigenvector $I_{N}$. The remaining eigenvalues are one with multiplicity $N-1$. Hence, $e(t)=0$ if and only if $w_{i} \eta_{i}(t)=w_{j} \eta_{j}(t)$, for all $i, j=1,2, \ldots, N$. Convergence of $e(t)$ to zero ensures $w_{i} \eta_{i}(t)=w_{j} \eta_{j}(t)$, which is equivalent to (Eq. 7). The other condition is the same as (Eq. 8).

Remark 3. In Lemma 2, we define an error $e_{i}(t)=w_{i} \eta_{i}(t)-\frac{1}{N} \sum_{j=1}^{N} w_{j} \eta_{j}(t)$, the convergence of which leads to an optimal solution of (Eq. 3). Conventionally, when $w_{i}=w_{j}=1$ with $i, j=1,2, \ldots, N$, the error $e_{i}(t)=\eta_{i}(t)-$ $\frac{1}{N} \sum_{j=1}^{N} \eta_{j}(t)$ is applied for complete consensus in ICs; however, the proposed weighted EDP does not require the complete consensus, rather it needs a different sign-consensus treatment between ICs of DGs, which means that the signs of ICs of DGs should have a consensus. Thanks to the recently investigated study on sign-consensus (Jiang et al., 2017), which can be applied as a tool to achieve optimal solution in the present case.

\section{Proposed Distributed Optimization Approach}

We take a balanced initial condition that can be achieved as seen in Xing et al. (2019), Yu et al. (2020), and references therein.

$$
\sum_{i=1}^{N} P_{G_{i}}(0)=P_{D}
$$

Assumption 1. The bidirectional graph $\mathcal{G}$ is strongly connected. The proposed optimization protocol has the form

$$
\left\{\begin{array}{l}
\dot{\eta}_{i}(t)=2 c \gamma_{i} \sum_{j=1}^{N} H_{i j} a_{i j}\left(w_{i} \eta_{i}(t)-w_{j} \eta_{j}(t)\right), \\
H_{i j}=\left\{\begin{array}{cl}
\frac{1}{a_{i j}}, & \text { if } a_{i j} \neq 0, \\
0, & \text { if } a_{i j}=0,
\end{array}\right.
\end{array}\right.
$$

where $c$ is a coupling weight and $H_{i j}$ is used to normalized the entries $a_{i j}$. This normalization can be helpful for normalizing the large or small strengths of connection between nodes. Furthermore, we can have signed graphs with negative entries of $a_{i j}$. Practically, signed graphs can appear due to transmitting information with amplification through inverting amplifiers. In such a case, gains $H_{i j}$ are necessary for normalizing the weights. Owing to the modification of $H_{i j}$, the presented approach can be applied to signed graphs. Each generator $i$ can update its IC, $\eta_{i}(t)$, by using its own weighted IC, $w_{i} \eta_{i}(t)$, and the weighted IC, $w_{j} \eta_{j}(t)$, of neighboring generator.

Remark 4. The proposed distributed optimization protocol has two distinctions compared to the existing methods (Xing et al., 2019; Yu et al., 2020; Chen et al., 2019). First, the IC of a generator can be updated based on local information of weighted IC (rather than IC), which is applied to resolve weighted EDP (Eq. 3). Second, we have introduced a normalization factor $H_{i j}$, through which the proposed approach can be applied to bidirectional (even signed) graphs, while the existing studies are limited to the conventional graphs. Note that in a realistic communication scenario, two generators can share weighted ICs with different strengths, leading to a bidirectional connection. The connection strength can also be negative due to use of inverting amplifiers, often used in signal transmissions.

The following theorem provides the optimal solution of (Eq. 3).

Theorem 1. Consider the $N$ generators satisfying Assumption 1 and validating (Eq. 16). The distributed protocol (Eq. 17) for $c>$ 0 ensures convergence of $P_{G_{i}}$ to the optimal solution $P_{G_{i}}^{\star}$ for the constrained weighted EDP (Eq. 3).

Proof. Selecting $\phi=\sum_{j=1}^{N} w_{j} \eta_{j}(t)$, we have

$$
\begin{gathered}
w_{i} \eta_{i}(t)-w_{j} \eta_{j}(t)=w_{i} \eta_{i}(t)+\phi-\phi-w_{j} \eta_{j}(t) \\
=e_{i}(t)-e_{j}(t) .
\end{gathered}
$$

Putting (Eq. 18) in (Eq. 17) obtains

$$
\dot{\eta}_{i}(t)=2 c \gamma_{i} \sum_{j=1}^{N} H_{i j} a_{i j}\left(e_{i}(t)-e_{j}(t)\right) .
$$

Taking the time-derivative of (Eq. 14) leads to

$$
\dot{e}_{i}(t)=w_{i} \dot{\eta}_{i}(t)-\frac{1}{N} \sum_{j=1}^{N} w_{j} \dot{\eta}_{j}(t) .
$$

Using (Eq. 19) into (Eq. 20), we have 


$$
\begin{aligned}
\dot{e}_{i}(t)= & 2 c \gamma_{i} w_{i} \sum_{j=1}^{N} H_{i j} a_{i j}\left(e_{i}(t)-e_{j}(t)\right) \\
& -\frac{2 c}{N} \sum_{j=1}^{N} \gamma_{j} w_{j} \sum_{i=1}^{N} H_{j i} a_{j i}\left(e_{j}(t)-e_{i}(t)\right) .
\end{aligned}
$$

The last term of Eq. 21 can be evaluated as

$$
\sum_{j=1}^{N} \gamma_{j} w_{j} \sum_{i=1}^{N} H_{j i} a_{j i}\left(e_{j}(t)-e_{i}(t)\right)=0 .
$$

Substituting (Eq. 22) into (Eq. 21) leads to

$$
\dot{e}_{i}(t)=2 c \gamma_{i} w_{i} \sum_{j=1}^{N} H_{i j} a_{i j}\left(e_{i}(t)-e_{j}(t)\right) .
$$

For a positive scalar $p$, consider a Lyapunov function as

$$
V\left(e_{i}(t)\right)=0.25 \sum_{i=1}^{N} \gamma_{i}^{-1} w_{i}^{-1} e_{i}^{2} .
$$

Taking the time-derivative of (Eq. 24), we obtain

$$
\dot{V}\left(e_{i}(t)\right)=0.5 \sum_{i=1}^{N} \gamma_{i}^{-1} w_{i}^{-1} e_{i} \dot{e}_{i} .
$$

From Eq. 23, Eq. 25 becomes

$$
\dot{V}\left(e_{i}(t)\right)=c \sum_{i=1}^{N} e_{i} \sum_{j=1}^{N} H_{i j} a_{i j}\left(e_{i}(t)-e_{j}(t)\right) .
$$

Under Assumption 1, we have $H_{i j} a_{i j}=H_{j i} a_{j i}$. Let us define a symmetric matrix as $\tilde{\mathcal{L}}=\left[\tilde{l}_{i j}\right]_{N \times N}$, where

$$
\tilde{l}_{i j}= \begin{cases}-H_{i j} a_{i j}, & i \neq j, \\ \sum_{j=1}^{N} H_{i j} a_{i j}, & i=j .\end{cases}
$$

Using the information of normalized Laplacian into (Eq. 26) leads to

$$
\dot{V}\left(e_{i}(t)\right)=-c e^{T}(t) \tilde{\mathcal{L}} e(t) \leq-c e^{T}(t) \alpha(\tilde{\mathcal{L}}) e(t)<0 .
$$

As $\dot{V}\left(e_{i}(t)\right)<0$, the convergence of $e_{i}(t)$ to the origin is attained, that is to say, the first condition in Lemma 2 holds. To consider the second condition, we apply the analysis of (Eq. 17). Under $\bar{\gamma}=\sum_{j-1}^{N}\left(2 \gamma_{j}\right)^{-1}$, dividing Eq. 17 by $\bar{\gamma} \gamma_{i}$, we obtain

$$
\frac{1}{\gamma_{i} \bar{\gamma}} \dot{\eta}_{i}(t)=\frac{c}{\bar{\gamma}} \sum_{j=1}^{N} H_{i j} a_{i j}\left(w_{i} \eta_{i}(t)-w_{j} \eta_{j}(t)\right) \text {. }
$$

Let us assign $\Psi=\sum_{i=1}^{N} \frac{1}{\gamma_{i}} \eta_{i}(t)$, its derivative along (Eq. 29) becomes

$$
\dot{\Psi}=\frac{c}{\bar{\gamma}} \sum_{i=1}^{N} \sum_{j=1}^{N} H_{i j} a_{i j}\left(w_{i} \eta_{i}(t)-w_{j} \eta_{j}(t)\right) .
$$

Note that $\sum_{i=1}^{N} \sum_{j=1}^{N} H_{i j} a_{i j}\left(w_{i} \eta_{i}(t)-w_{j} \eta_{j}(t)\right)=0$; therefore, $\dot{\Psi}=0$. It further implies that there exists a constant $\chi$ such that $\Psi$ $=\chi$. Consequently, we have

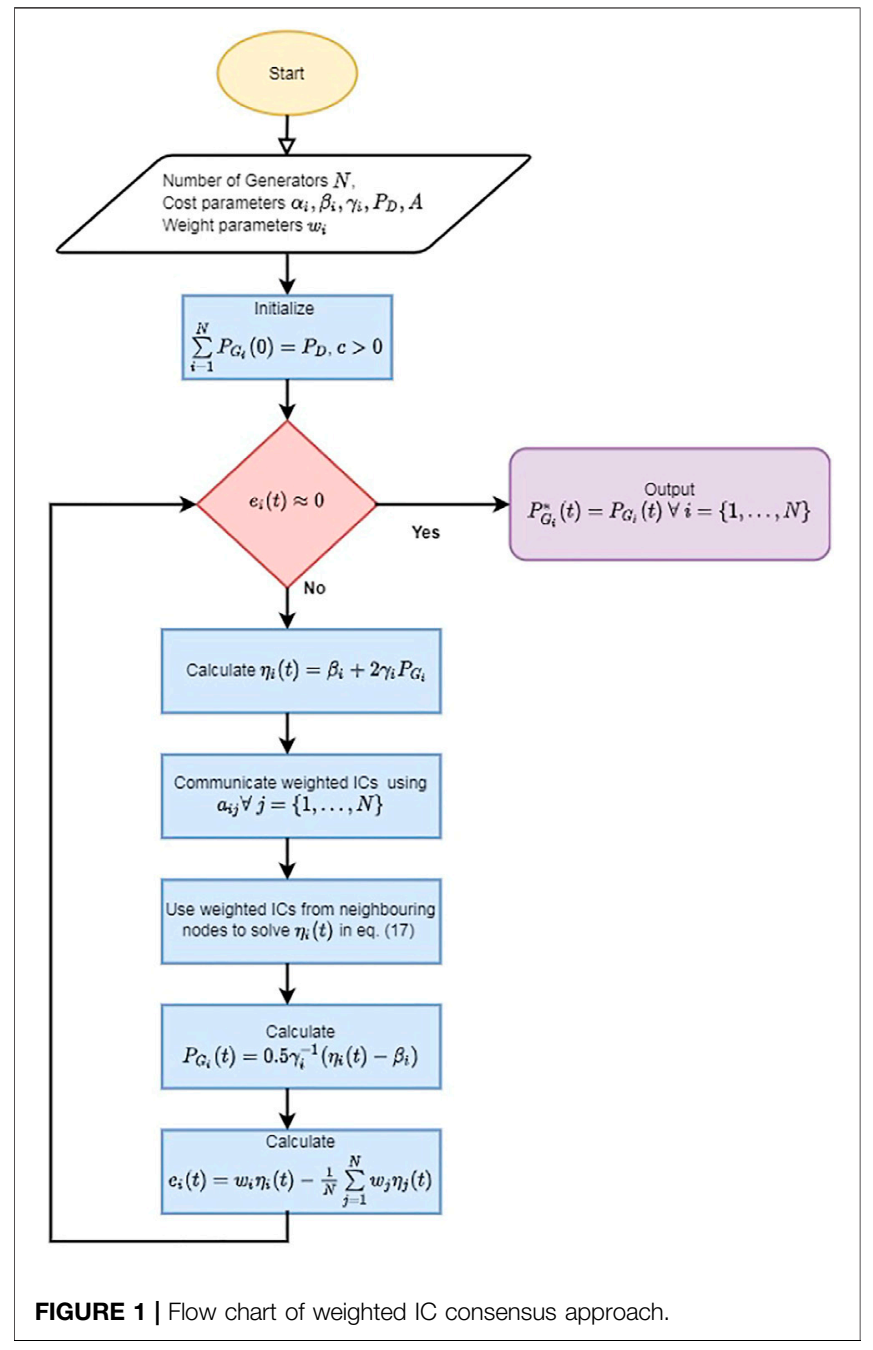

$$
\sum_{i=1}^{N} \frac{1}{\gamma_{i} \bar{\gamma}} \eta_{i}(t)=\chi .
$$

which by substituting (Eq. 6) implies

$$
\sum_{i=1}^{N}\left(\frac{\beta_{i}}{2}+P_{G_{i}}(t)\right)=2 \bar{\gamma} \chi .
$$

For investigating total generation from (Eq. 32), we have

$$
\sum_{i=1}^{N} P_{G_{i}}(t)=2 \bar{\gamma} \chi-\frac{1}{2} \sum_{i=1}^{N} \beta_{i}=P_{D} .
$$

It shows that the second condition of convergence of $\sum_{i=1}^{N} P_{G_{i}}$ to $P_{D}$ is also attained. By the application of Lemma 2, $P_{G_{i}}$ will converge to $P_{G_{i}}^{*}$, which completes the proof.

Although the presented approach in Theorem 1 provides the convergence condition, the rate of convergence has not been investigated in the presented study. Here we provide guidelines to improve the convergence rate. By selecting the larger control parameter $c$ in (Eq. 17), fast convergence of the proposed 
Input: Number of generators $N$, Cost parameters of generators $\left(\alpha_{i}, \beta_{i}\right.$,

$\left.\gamma_{i}, A, P_{D}\right)$, Weight parameters $\left(w_{i}\right) \forall i=\{1, \ldots, N\}$.

Output: $P_{G_{i}}^{*} \forall i=\{1, \ldots, N\}$ such that $\sum_{i=1}^{N} P_{G_{i}}^{*}(t)=P_{D}$.

Initialize: $\sum_{i=1}^{N} P_{G_{i}}(0)=P_{D}, c>0$

while $e_{i}(t) \neq 0 \forall i=\{1, \ldots, N\}$ do

for $i=1,2, \ldots, N$ do

Calculate IC $\eta_{i}(t)$ using equation (6) based on $P G_{i}$ in the previous iteration.

Communicate weighted ICs based on topology $a_{i j} \forall j=\{1, \ldots, N\}$.

Use weighted ICs from neighbouring nodes to solve $\eta_{i}(t)$ in equation (17).

Use equation (6) to calculate $P G_{i}$.

end for

Calculate $e_{i}(t)$ from equation (14).

end while

return $P G_{i}$

approach can be attained. However, careful attention should be accounted in selecting the larger gain, as it can cause sensitivity of the algorithm against noises by amplifying their effects. The convergence of the protocol (Eq. 17) can also be faster for the large value of $\alpha(\tilde{\mathcal{L}})$, which can be increased by having more edges (connections between nodes) in a graph topology.

The flowchart of the proposed weighted IC consensus approach is shown in Figure 1. After initialization, the value of IC at each node is calculated based on $P_{G_{i}}$ at the previous iteration. Subsequently, the nodes communicate their IC values to the connected node, where the connections among nodes are defined through an adjacency matrix. A new value of IC and $P_{G_{i}}$ is calculated by the node based on the weighted ICs from the nodes. Finally, a check is introduced to continuously monitor the convergence of weighted ICs that will ensure the optimal values of $P G_{G_{i}}$, i.e., $P_{G_{i}}^{*}$. The algorithmic form of the distributed optimization approach for weighted IC consensus is also given in Algorithm 1.

Remark 5. Theorem 1 provides the main optimization results for resolving the weighted EDP (Eq. 3). To the best of our knowledge, a distributed optimization approach for solving weighted EDP (Eq. 3) has been provided for the first time. The results are attained by the application of a different sign-consensus theory along with the generation and weighted IC dynamical analysis. The proposed approach is also advantageous as it can be applied to attain several constraints in a simple manner by adjusting weights. The consensus control approaches like Sun et al. (2015) consider the attainment of agents' same behavior by applying feedback. While the consensus-based optimization methods can be considered the progress of these control approaches for a distributed optimization, an objective function is optimized, like the cost of generation is optimized, and constraints like generation constraint.
Remark 6. In a network, electrical losses over transmission lines can be a challenging problem, which should be considered for a realistic EDP problem. Let $P_{L}$ be an estimate of the electrical losses in the transmission lines, then we require $P_{D}+P_{L}-\sum_{i=1}^{N} P_{G_{i}}=0$, instead of (Eq. 13). This condition can be attained by simply using the modified initial condition as $\sum_{i=1}^{N} P_{G_{i}}(0)=P_{D}+P_{L}$.

Remark 7. Recently, economic dispatch has been addressed in Zhao et al. (2018) and Shi et al. (2020) for saving the communication bandwidth through an even-triggering mechanism. Another interesting study of Shi et al. (2021) has developed an optimization protocol for exponential convergence. Compared to these results, the presented approach considers an optimization problem by considering a different weighted cost function. Based on the studies Zhao et al. (2018) and Shi et al. (2020), the present approach can be extended for event-triggered communication, rather than the time-triggered approach, for the efficient bandwidth utilization. Furthermore, the presented approach can be extended for exponential rate of the optimization, based on the results of Shi et al. (2021), for controlling the convergence rate parameter.

\section{EXTENSION TO OPF PROBLEM}

OPF is an advanced optimization problem in micro-grids, the aim of which is to minimize the cost of generated power under EDP constraints along with the practical constraints, like bus voltage limits, bus power limits, and bus losses. Compared with EDP, OPF considers the cost minimization along with implementation aspects of power flow in a grid. Generally, OPF is a complex problem due to 1) additional nonlinear constraints, 2) nonconvexity of the new constraints, 3) requirement of more 
TABLE 1 | Parameters for IEEE-30 bus system.

\begin{tabular}{cccccc}
\hline $\boldsymbol{i}$ & $\boldsymbol{\alpha}_{\boldsymbol{i}}$ & $\boldsymbol{\beta}_{\boldsymbol{i}}$ & $\boldsymbol{y}_{\boldsymbol{i}}$ & $\mathbf{P}_{\mathbf{G}_{\mathbf{i}}^{\mathbf{m}}}$ & $\mathbf{P}_{\mathbf{G}_{\mathbf{i}}}^{\mathbf{M}}$ \\
\hline 1 & 0 & 2 & 0.00375 & 50 & 200 \\
2 & 0 & 1.75 & 0.0175 & 20 & 80 \\
3 & 0 & 1 & 0.0625 & 15 & 50 \\
4 & 0 & 3.25 & 0.00834 & 10 & 35 \\
5 & 0 & 3 & 0.025 & 10 & 30 \\
6 & 0 & 3 & 0.025 & 12 & 40 \\
\hline
\end{tabular}

computational resources, and 4) uncertain environment in largescale systems, as observed in Pourbabak et al. (2019). In addition, attainment of a distributed OPF solution for a distributed optimization issue further complicates this problem, as seen in Pourbabak et al. (2019), Pourbabak et al. (2020), Liu et al. (2017), Yun Liu et al. (2019), Linfeng Yang et al. (2020), and Wang et al. (2017).

Here, we modify the proposed EDP approach in Theorem 1 for OPF by the application of the recent results in Pourbabak et al. (2020) for the weighted cost function, based on weighted IC consensus. Let $N_{d l}, N_{g}, N_{\text {load }}$, and $N_{b}$ represent the sets of distributed lines, generators, load lines, and all lines over a DC micro-grid. For active generation $P_{G_{i}}$ and demand $P_{D_{i}}$ of $i$ th generator, the OPF problem can be represented as

$$
\min C_{w t}\left(P_{G_{i}}\right)=\sum_{i=1}^{N} w_{i} C_{i}\left(P_{G_{i}}\right),
$$

such that

$$
\begin{gathered}
\sum_{i=1}^{N} P_{D_{i}}=\sum_{i=1}^{N} P_{G_{i}}, \\
P_{n e t, i}=\left\{\begin{array}{cc}
P_{G_{i}}-P_{D_{i}}, & \forall i \in\left(N_{g}+N_{l}\right), \\
0, & \forall i \notin\left(N_{g}+N_{l}\right),
\end{array}\right. \\
P_{n e t, i}=\sum_{n \in\left(N_{b}-i\right)}^{N} P_{i n}, \forall i \in N_{b}, \\
v_{i}^{\min } \leq v_{i} \leq v_{i}^{\max }, \forall i \in N_{g} \\
P_{\text {in }}=G_{i n}\left(v_{i} v_{i}-v_{i} v_{n}\right), \forall i, n \in N_{b}, \\
P_{\text {in }} \leq P_{i n}^{\max }, \forall i, n \in N_{d l},
\end{gathered}
$$

where $v_{i}$ denotes the $i$ th generator bus voltage with minimum and maximum limits as $v_{i}^{\min }$ and $v_{i}^{\max }$, respectively. $G_{i n}$ represents the conductance of a line between buses $i$ and $n$. $P_{n e t, i}$ shows the net power injected in $i$ th bus. The power flow between $i$ and $n$ buses is represented by $P_{i n}$ with limit $P_{i n}^{\max }$. The discretized first-order approximation form of (Eq. 17) with sample time $T_{s}$ is given as

$$
\left\{\begin{array}{l}
\eta_{i}(j, k+1)=-\eta_{i}(j, k)+2 c \gamma_{i} T_{s} \sum_{j=1}^{N} H_{i j} a_{i j}\left(w_{i} \eta_{i}(j, k)-w_{j} \eta_{j}(j, k)\right), \\
H_{i j}= \begin{cases}\frac{1}{a_{i j},} & \text { if } a_{i j} \neq 0, \\
0, & \text { if } a_{i j}=0 .\end{cases}
\end{array}\right.
$$

Upon convergence of $\eta_{i}(j, k+1) \rightarrow \eta_{c, i}(j)$ from (Eq. 35), we can determine the generations as

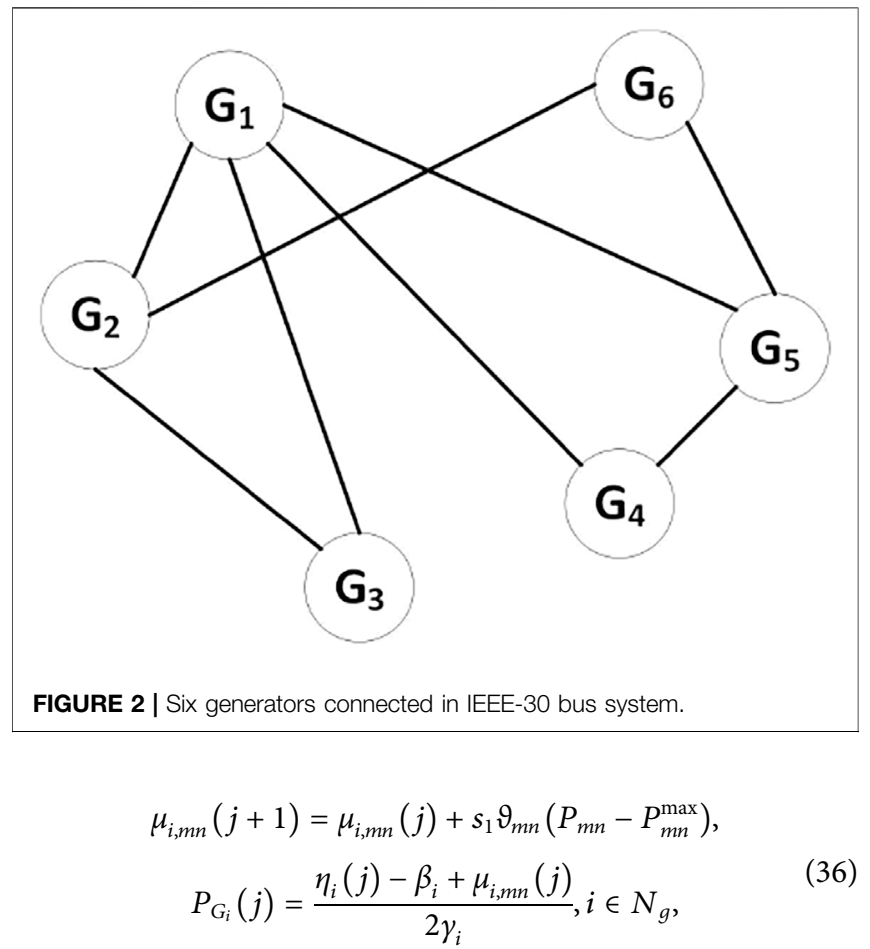

where $s_{1}$ is a small value and $\vartheta_{m n}$ shows sensitivity between $m$ and $n$ buses. Here, $\mu_{i, m n}(j)$ is the state for controlling the power flow limit between two buses. Let us assign $X_{i n}=v_{i} v_{n}$, the following approach for small scalars $s_{2}$ and $s_{3}$ can be used for validating the practical constraints through OPF (see details in Pourbabak et al. (2020)):

$$
\begin{aligned}
& X_{i n}(j+1)=X_{i n}(j)+s_{2}\left(X_{i i}(j) X_{n n}(j)-X_{i n}(j)\right), \\
& \forall i \in N_{b}, n \in\left(N_{b}-\{i\}\right), \\
& X_{i i}(j+1)=\left(P_{G_{i}}(j)-P_{D_{i}}+\sum_{n \in\left(N_{b}-\{i\}\right)}^{N} G_{i n} X_{i n}(j)\right)\left(\sum_{n \in\left(N_{b}-\{i\}\right)}^{N} G_{i n}\right)^{-1}, \\
& \eta_{i}(j+1, k)=\eta_{c, i}(j)+s_{3} \\
& \left(P_{G_{i}}(j)-P_{D_{i}}(j)-\sum_{n \in\left(N_{b}-\{i\}\right)}^{N} G_{i n}\left(X_{i i}(j)-X_{i n}(j)\right)\right), \forall i \in N_{b} .
\end{aligned}
$$

The OPF problem for $\min C_{w t}\left(P_{G_{i}}\right)=\sum_{i=1}^{N} w_{i} C_{i}\left(P_{G_{i}}\right)$ under (Eq. 34) can be resolved by the application of (Eqs 35-39) for a new weighted cost function. In the future, the detailed analysis of such an algorithm along with the practical model for OPF for analyzing the results can be investigated.

Remark 8. The conventional EDP methods (Xing et al., 2019; Yu et al., 2020; Chen et al., 2019) cannot be applied practically to resolve the OPF issue in a distributed way. There are very limited distributed methods, as seen in Pourbabak et al. (2019), Pourbabak et al. (2020), Liu et al. (2017), Yun Liu et al. (2019), Linfeng Yang et al. (2020), and Wang et al. (2017), which deal with the OPF problem over a micro-grid using distributed algorithms. It is worth mentioning that the 


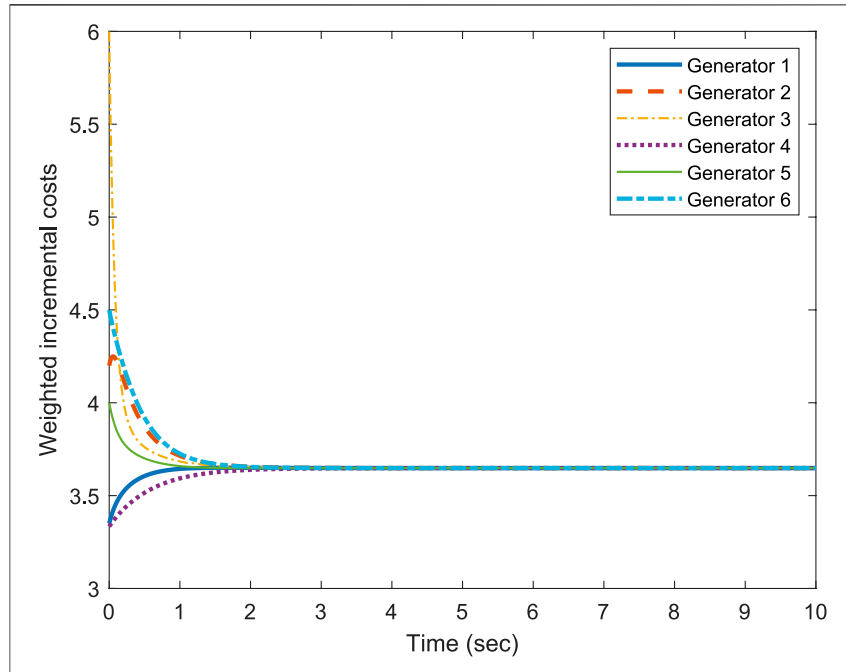

FIGURE 3 | Weighted ICs of individual generators versus time (sec) under the conventional approach (unity weights).

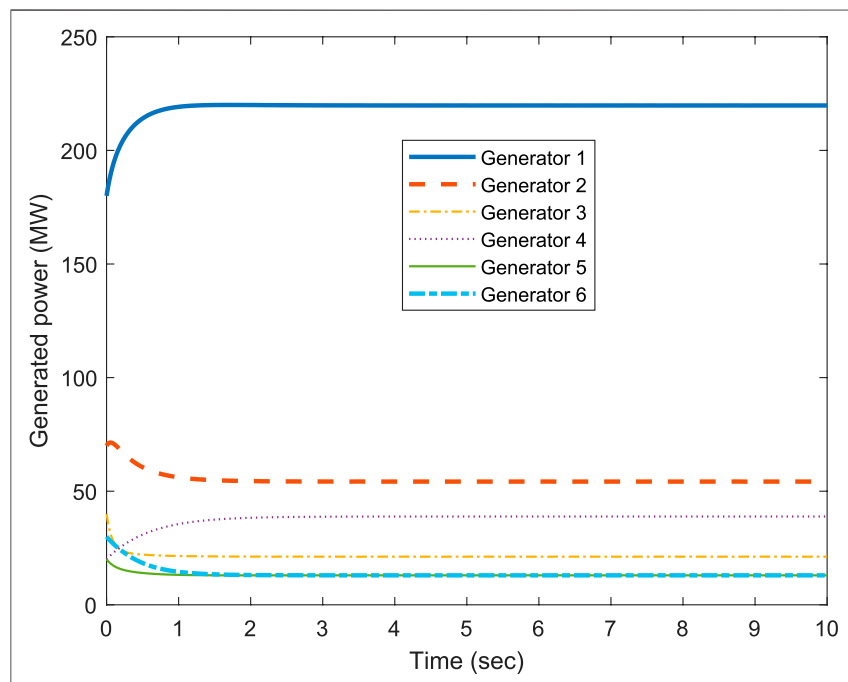

FIGURE 4 | Power generations (MW) of individual generators versus time (sec) under the conventional approach (unity weights).

proposed approach in Theorem 1 has been extended for a distributed OPF protocol by considering these exceptional works.

\section{SIMULATION RESULTS}

Here, we provide the simulation results of the proposed optimization methodology for the IEEE-30 bus system and for a large-scale IEEE-118 bus system. The IEEE-30 bus system is selected to emphasize basic understanding of the proposed technique and for the comparison of our approach with the existing methods. The IEEE-118 bus system has been adapted to test the application of the proposed approach to a complex system

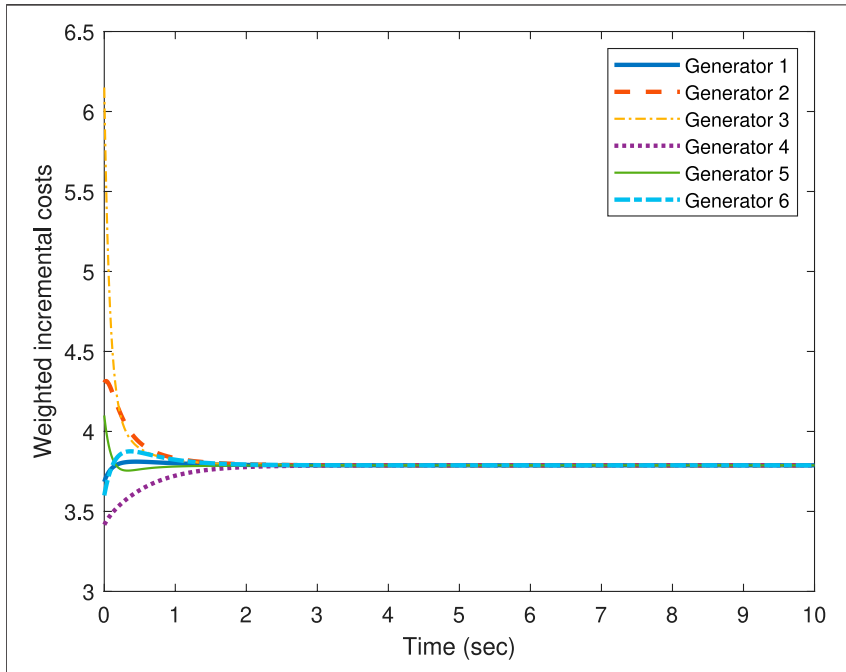

FIGURE 5 | Weighted ICs of individual generators versus time (sec) using the proposed approach (via weight selection), $w_{1}=1.1, w_{2}=w_{3}=w_{4}=w_{5}=$ 1.025 and $w_{6}=0.8$.

and to investigate adjustment in the resultant method for the application to a large-scale system.

\section{Application to IEEE-30 Bus System}

We consider IEEE-30 bus system (He et al., 2019) for testing the proposed weighted EDP approach, which consists of six distributed generators. The simulations are carried out in MATLAB environment, motivated by the approaches of Wang et al. (2021) and Hu et al. (2021), by considering a total demand of $P_{D}=360 \mathrm{MW}$. The parameters are taken as $\alpha_{i}=0$ for all generators, $\beta_{i}$ are given by $2,1.75,1,3.25,3$, and 3 , and $\gamma_{i}$ are taken as $0.00375,0.0175,0.0625,0.00834,0.025$, and 0.025 , for $i=1,2, \ldots, 6$, respectively. The minimum and maximum generation constraints are given by sets $\{50,20,15,10,10,12\}$ and $\{200,80,50,35,30,40\}$. All the cost coefficients of IEEE-30 bus system are shown in Table 1. The graph between DGs of IEEE-30 bus system is shown in Figure 2 (Lewis et al., 2014). The location of generators and the connection between DGs can be represented via an adjacency matrix. The adjacency matrix $\mathcal{A}$ has the form

$$
\mathcal{A}=\left[\begin{array}{llllll}
0 & 1 & 1 & 1 & 1 & 0 \\
1 & 0 & 1 & 0 & 0 & 1 \\
1 & 1 & 0 & 0 & 0 & 0 \\
1 & 0 & 0 & 0 & 1 & 0 \\
1 & 0 & 0 & 1 & 0 & 1 \\
0 & 1 & 0 & 0 & 1 & 0
\end{array}\right]
$$

All the simulations are carried out in MATLAB. The initial conditions for the generation are taken as $P_{G_{1}}(0)=180 \mathrm{MW}$, $P_{G_{2}}(0)=70 \mathrm{MW}, P_{G_{3}}(0)=40 \mathrm{MW}, P_{G_{4}}(0)=20 \mathrm{MW}, P_{G_{5}}(0)=$ $20 \mathrm{MW}$, and $P_{G_{5}}(0)=30 \mathrm{MW}$. In the following, we first apply the conventional approach by taking $w_{i}=1 \forall i=\{1,2, \ldots, 6\}$. Afterward, we show the effectiveness of appropriate weight selection in dealing with the capacity and environmental 


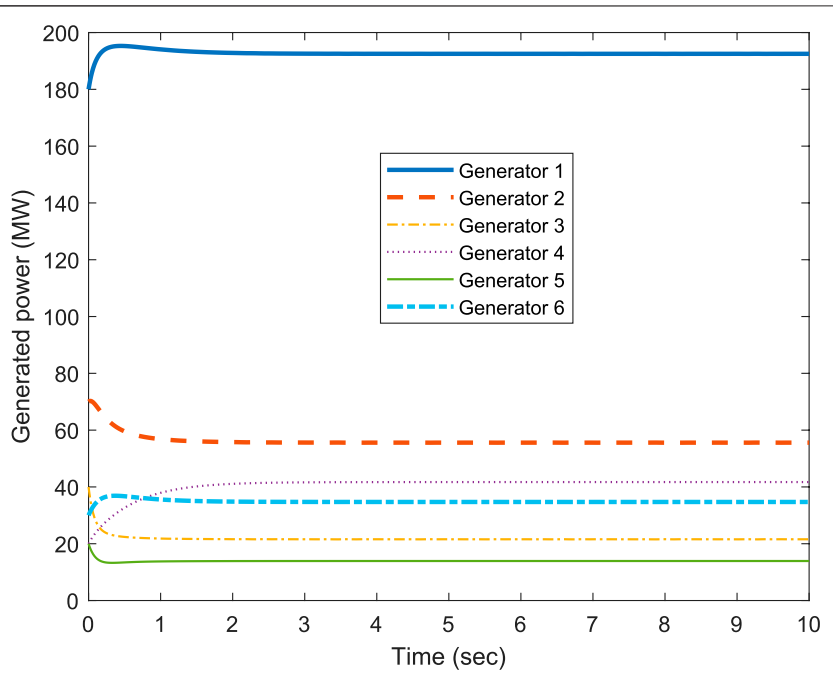

FIGURE 6 | Power generations (MW) of individual generators versus time (sec) using the proposed approach (via weight selection), $w_{1}=1.1, w_{2}=$ $w_{3}=w_{4}=w_{5}=1.025$ and $w_{6}=0.8$.

constraints. By solving Theorem 1 for unity weights, we have selected $c=100$.

Figure 3 shows the results of weighted ICs for a special case, in which $w_{1}=w_{2}=w_{3}=w_{4}=w_{5}=w_{6}=1$. This special scenario is equivalent to the conventional IC consensus scenario depicted in Xing et al. (2019), Yu et al. (2020), and Chen et al. (2019) without generation constraints. The IC curves of all the generators converge rapidly to a common value. For the unity weights, the corresponding generation plots are shown in Figure 4. The results are optimal for $C_{t}\left(P_{G_{i}}\right)$; however, generator $G_{1}$ crosses the upper limit of $200 \mathrm{MW}$, violating the constraints in (Eq. 4). Also note that the power generation of $G_{5}$ and $G_{6}$ converges to $20 \mathrm{MW}$. As mentioned earlier that the existing methods (Xing et al., 2019; Yu et al., 2020; Chen et al., 2019) are special case of the proposed approach in terms of weights, the same violation of the capacity constraint while achieving IC consensus can be observed. In addition, if $G_{6}$ is environmental friendly, we cannot increase its generation under reduced cost using the existing distributed methods.

For the verification of the proposed method, we assume that the generator $G_{6}$ is environment friendly. Therefore, we increase its generation in addition to (Eq. 4) for $G_{1}$. For that, we can select our weights $w_{i}$ (which is not possible in conventional approaches). Let us choose $w_{1}=1.1, w_{2}=w_{3}=w_{4}=w_{5}=$ 1.025 and $w_{6}=0.8$. The weight $w_{1}$ is assigned a higher value to reduce $G_{1}$ generation below $200 \mathrm{MW}$. The smaller value of $w_{6}$ is assigned to increase the generation of $G_{6}$ due to its environment friendly nature. Under these weights, the results of the proposed approach for weighted ICs and generations are demonstrated in Figure 5 and Figure 6. Clearly, the weighted IC consensus has been achieved as demonstrated in Figure 5. In addition, the steady-state generation for $G_{1}$ is around $192 \mathrm{MW}$, validating the capacity constraint, and for $G_{6}$, we are able to increase its generation to around $34 \mathrm{MW}$ by simply reducing the weight to 0.8 . Hence, the optimal solution to the proposed weighted EDP (Eq. 3) can be achieved through the proposed methods for validating additional constraints such as capacity and environment constraints.

Here, we compare the proposed approach in Theorem 1 with the existing methods of Krishnamurthy et al. (2017), McLarty et al. (2019), and Yu et al. (2015). The conventional approaches (Krishnamurthy et al., 2017) and (McLarty et al., 2019) are based on the central methods, for which data sharing with dispatch center is needed along with central computation. In contrast, the proposed approach applies the distributed computation of the optimal solution to attain a quick solution with several processors, installed at the generator levels, without sharing the data with a central unit. Hence, the distributed computation applied in the proposed method has a simple advantage over Krishnamurthy et al. (2017) and McLarty et al. (2019) for fast computation using distributed processors, based on first-order simple differential equations. In addition, the proposed method also avoids data transmission delays caused by data transmission from/to the central unit, and it ensures data confidentiality as well. The comparison of the proposed method

TABLE 2 | Comparison of the proposed method with the existing study.

\begin{tabular}{|c|c|c|c|}
\hline \multirow[t]{4}{*}{ Quantities } & \multirow[t]{4}{*}{ He et al. (2019) } & Theorem 1: $w_{1}=1.1$ & Theorem 1: $w_{2}=1$ \\
\hline & & $w_{2}=w_{3}=1.025$ & $w_{1}=w_{4}=1.1$ \\
\hline & & $w_{4}=w_{5}=1.025$ & $w_{3}=w_{5}=1$ \\
\hline & & $w_{6}=0.8$ & $w_{6}=0.8$ \\
\hline$P_{G_{1}}$ limits $\{50,200\}$ & $219.8 \mathrm{MW}$ & $192.5 \mathrm{MW}$ & 197.2 MW \\
\hline$P_{G_{2}}$ limits $\{20,80\}$ & $54.25 \mathrm{MW}$ & $55.59 \mathrm{MW}$ & $59.34 \mathrm{MW}$ \\
\hline$P_{G_{3}}$ limits $\{15,50\}$ & $21.19 \mathrm{MW}$ & $21.55 \mathrm{MW}$ & $22.62 \mathrm{MW}$ \\
\hline$P_{G_{4}}$ limits $\{10,35\}$ & $38.87 \mathrm{MW}$ & $41.71 \mathrm{MW}$ & $28.7 \mathrm{MW}$ \\
\hline$P_{G_{5}}$ limits $\{10,30\}$ & $12.97 \mathrm{MW}$ & $13.92 \mathrm{MW}$ & $16.53 \mathrm{MW}$ \\
\hline$P_{G_{6}}$ limits $\{12,40\}$ & $12.97 \mathrm{MW}$ & $34.71 \mathrm{MW}$ & $35.66 \mathrm{MW}$ \\
\hline Weighted IC value (currency unit/MW) & 3.648 & 3.788 & 3.827 \\
\hline Cost function used & $\sum_{i=1}^{N} C_{i}\left(P_{G_{i}}\right)$ & $\sum_{i=1}^{N} w_{i} C_{i}\left(P_{G_{i}}\right)$ & $\sum_{i=1}^{N} w_{i} C_{i}\left(P_{G_{i}}\right)$ \\
\hline Optimal cost function (currency unit) & 1,032 & 1,082 & 1,084 \\
\hline Total generation cost (currency unit) & 1,032 & 1,046 & 1,048 \\
\hline
\end{tabular}




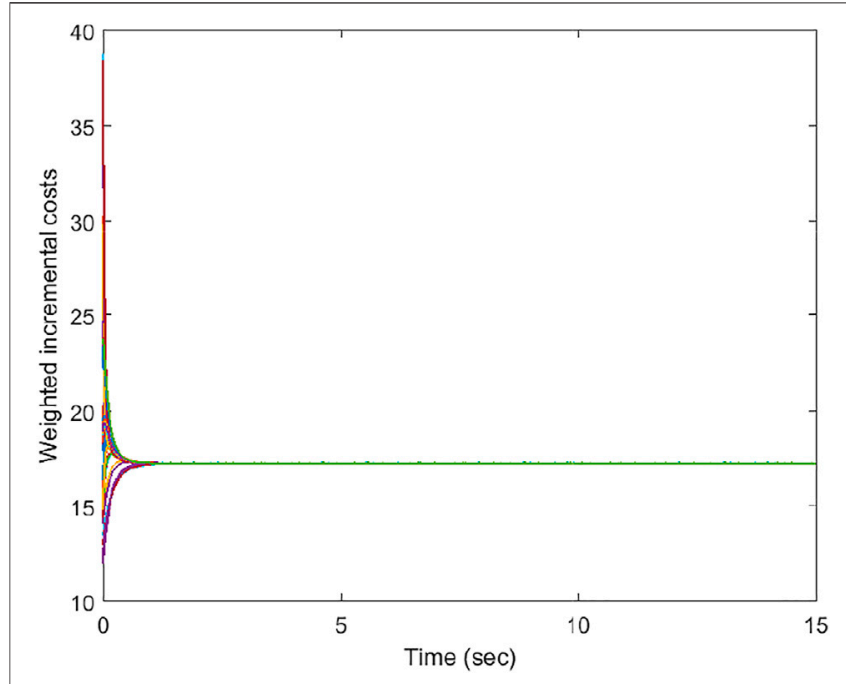

FIGURE 7 | IC consensus for unity weights of IEEE-118 bus system.

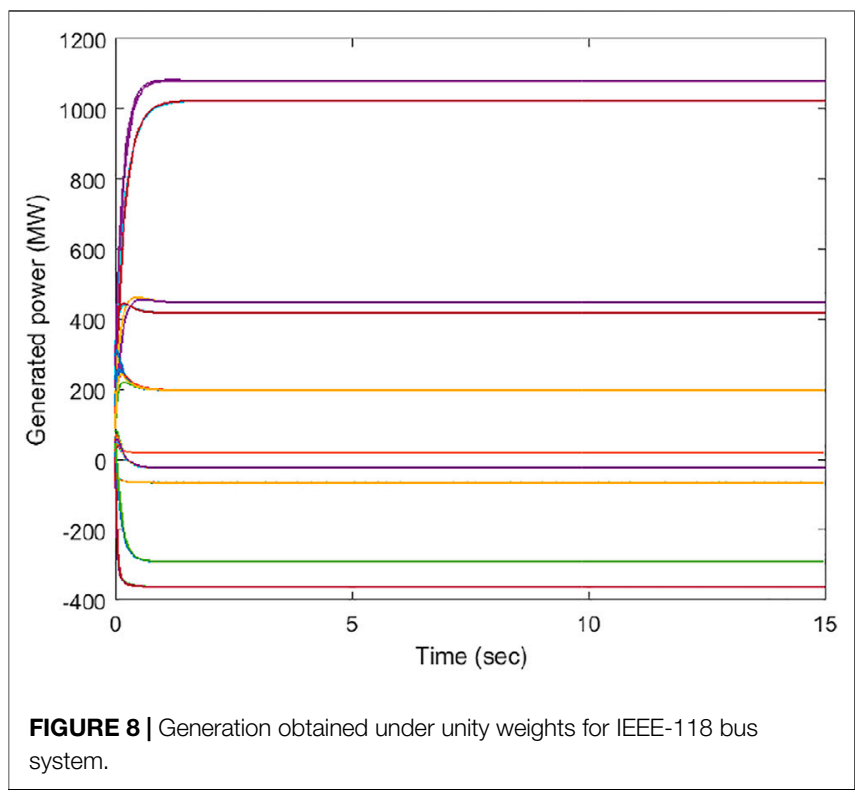

with Yu et al. (2015) [see also similar works (He et al., 2019; Xing et al., 2019; Yu et al., 2020), and (Chen et al., 2019) without weights] is provided in Table 2 for generations, cost functions, incremental costs, and total cost. For this purpose, the total demand is selected as $P_{D}=360 \mathrm{MW}$. First, we consider weights as $w_{1}=1.1, w_{2}=w_{3}=w_{4}=w_{5}=1.025$, and $w_{6}=0.8$ for the proposed method, as per the previous study. It can be observed that the proposed method respects the generation constraint of generator 1 , as $P_{G_{1}}$ satisfies the generation limits for the optimal solution. In addition, $P_{G_{6}}$ can be increased through the proposed method for increasing the generation of unit 6. Mainly, the proposed method has a different (weighted) cost function compared to He et al. (2019), which is capable of handling several generation constraints. The optimal solutions of

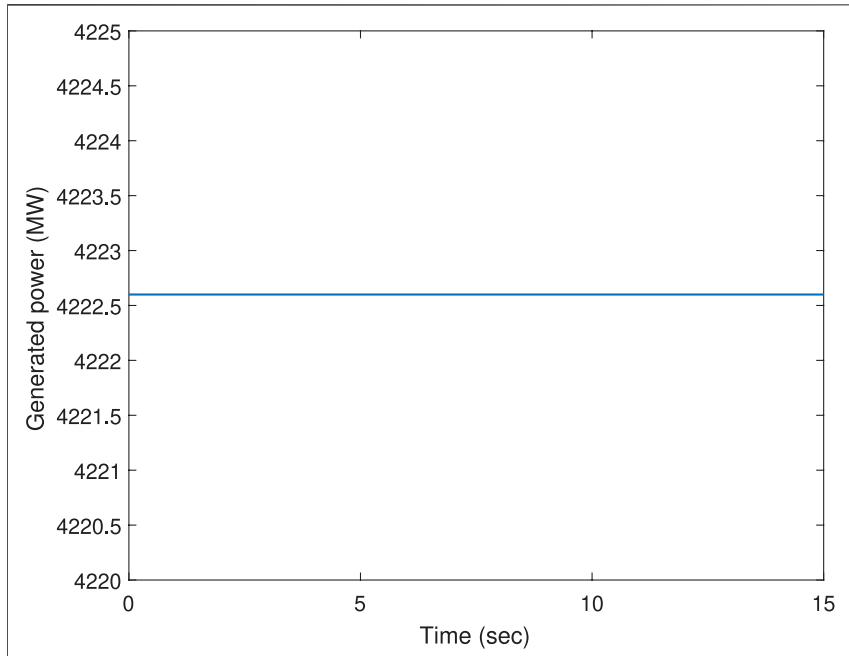

FIGURE 9 | Total generation under unity weights for IEEE-118 bus system.

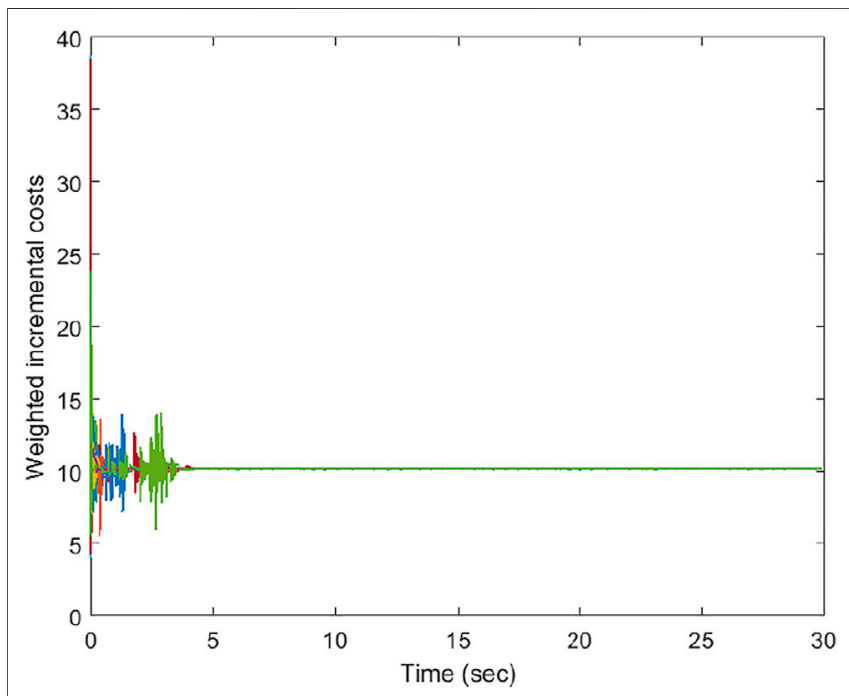

FIGURE 10 | Weighted IC consensus using the proposed approach for IEEE-118 bus system.

both cost functions are also provided herein. It should be noted that the total cost has been increased from 1,032 to 1,046 units. There is only $1.36 \%$ increase in the total generation cost, which is quite minimal for attaining the benefits handling the capacity and environmental constraints.

In the first experiment for weights $w_{1}=1.1, w_{2}=w_{3}=w_{4}=w_{5}$ $=1.025$ and $w_{6}=0.8$, the focus was on considering the generation units 1 and 6 , and their weights were considered to be different from the rest of the units. For this weight selection, it can be observed that the generation of unit 4 crosses the maximum limit, which was also observed in the original approach of $\mathrm{He}$ et al. (2019). To consider the capacity constraint for generator 4 , we have reconsidered the weights $w_{2}=1, w_{1}=w_{4}=1.1, w_{3}=w_{5}=1$, 


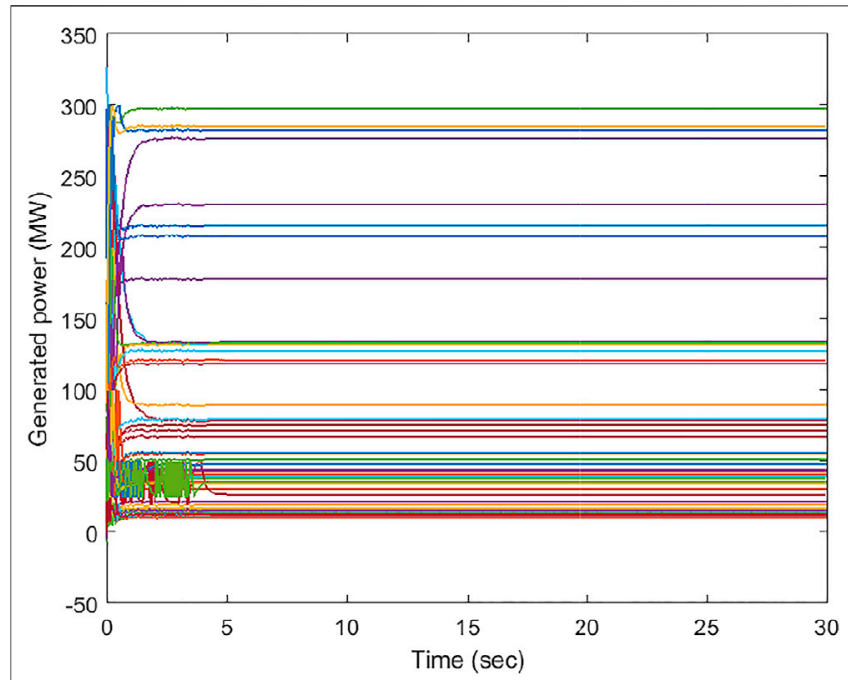

FIGURE 11 | Generation obtained using the proposed approach for IEEE-118 bus system.

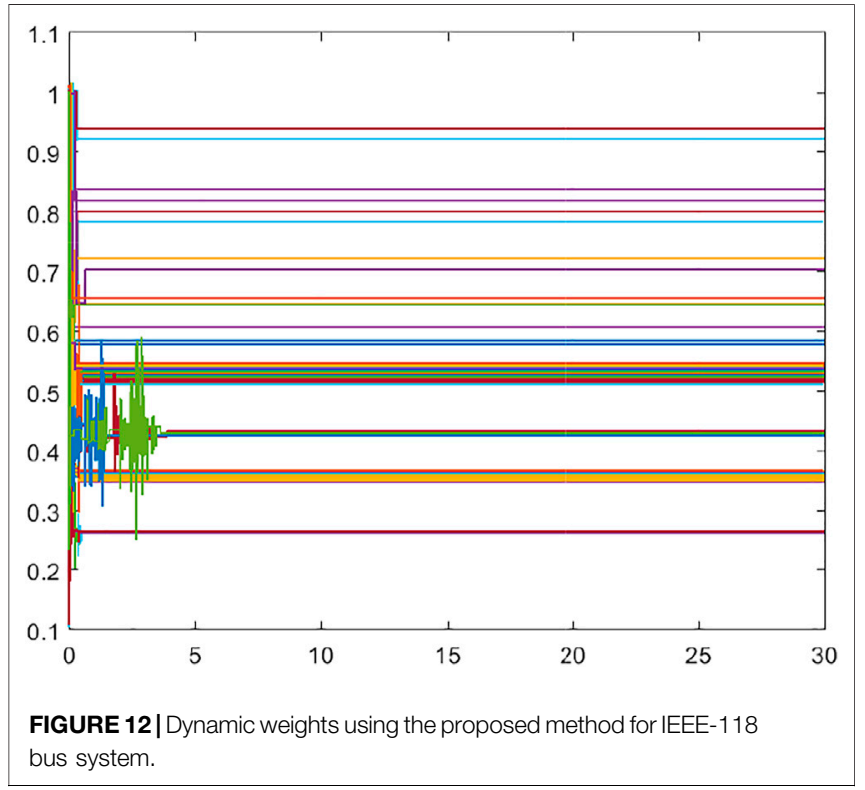

and $w_{6}=0.8$. For the increased weight of unit 4 , the capacity constraint is also validated as observed in the last column of Table 2 and as mentioned in Remark 1. It can be concluded that the weights can play a critical role in handling the network constraints. For further studies, we recommend a generationdependent weight selection via $w_{i}=\Upsilon_{i}\left(P_{G_{i}}\right)$, where the function $\Upsilon_{i}\left(P_{G_{i}}\right)$ can be taken as $\Upsilon_{i}\left(P_{G_{i}}\right)=1$ in the normal operating conditions, $\Upsilon_{i}\left(P_{G_{i}}\right)>1$ can be accounted for reducing the increase in generation, and $\Upsilon_{i}\left(P_{G_{i}}\right)<1$ can be chosen to limit the decrease in generation. Such a generation-dependent selection of weights through functions $\Upsilon_{i}\left(P_{G_{i}}\right)$ requires rigorous analysis and investigation, which can be considered in the future works.

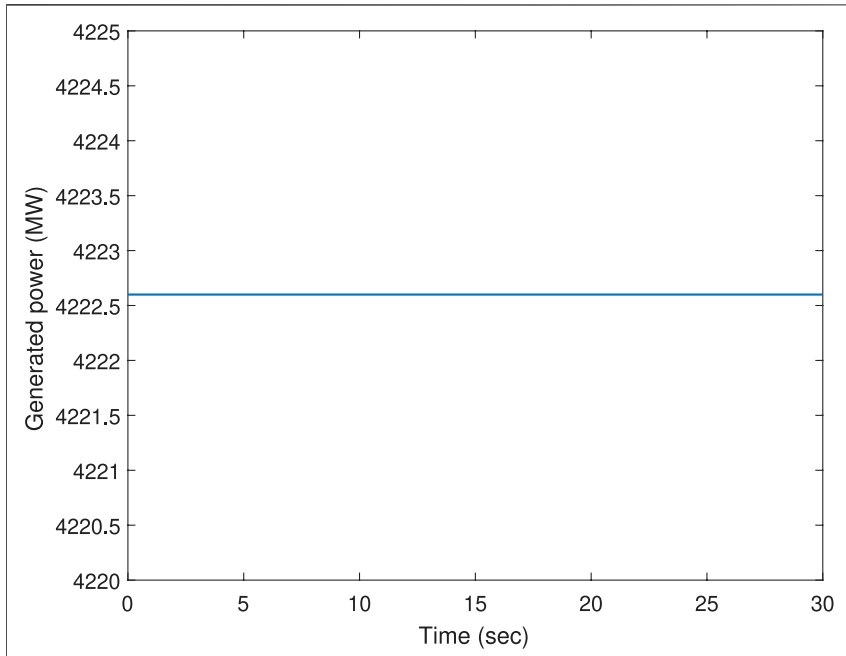

FIGURE 13 | Total generation using the proposed approach for IEEE118 bus system.

\section{Application to IEEE-118 Bus System}

IEEE-118 bus system is a 54 unit, 24 h system with 118 buses and 94 loads (IIT-Power-Group, 2003). The generators in this system are thermal units. It is a large-scale generation system and highly complex model owing to the involvement of constraints on several units. The values for parameters $\alpha_{i}, \beta_{i}, \gamma_{i}$, and minimum $P_{G_{i}}^{m}$ and maximum generation $P_{G_{i}}^{M}$ capacity are taken from IIT-Power-Group (2003). The range of values is given as follows: $\alpha_{i} \in\left[\begin{array}{ll}6.78 & 74.33\end{array}\right], \beta_{i} \in\left[\begin{array}{lll}8.3391 & 37.6968\end{array}\right]$, $\gamma_{i} \in\left[\begin{array}{ll}0.0024 & 0.0697\end{array}\right], P_{G_{i}}^{m} \in\left[\begin{array}{ll}20 & 150\end{array}\right]$, and $P_{G_{i}}^{M} \in\left[\begin{array}{ll}5 & 420\end{array}\right]$.

The system operates in three zones, and the parameters have been calculated on the basis of $24 \mathrm{~h}$ system at a fuel price of $1 \mathrm{USD} / \mathrm{MBtm}$. For our study, we consider the connections between generators as random to accommodate the stochastic nature of the algorithm, such that the graph remains undirected. The approach has been tested for a demand of $P_{D}=4,222.59 \mathrm{MW}$. Each connection strength is taken as either zero or unity. The initial generation conditions are also selected randomly based on uniform distribution. Owing to a large-scale system, it is challenging to design a distributed approach for such a system, ensuring the generation constraints.

If we select $w_{i}=1$ similar to the methods (Yu et al., 2015) (see also similar works (He et al., 2019; Xing et al., 2019; Yu et al., 2020), and (Chen et al., 2019) without weights), the approach in Theorem 1 provides the relevant graphs in Figure 7, Figure 8, and Figure 9. Figure 7 shows that the IC consensus has been achieved, and Figure 9 demonstrates that the required initial demand does not alter. However, the diagram of Figure 8 reveals that the generation values are beyond the upper or lower limits for many generators. Such a solution cannot be employed for the EDP. The existing methods suggest the saturation of generations (limiting to $P_{G_{i}}^{M}$ ); however, it can reduce the total generation without meeting the demand. The IEEE-118 bus system is a complex system and the results for a distributed handling are not easy for a real-world situation. 
To handle this dilemma, we consider two approaches 1) inclusion of weights $w_{i}$ and 2) updating weights $w_{i}$ dynamically. The updating of weights can be more meaningful for a complex system like IEEE-118 bus system. The weights are updated dynamically such that if we are going close to the upper limit of a generator, the corresponding weight can be increased, and vice versa. The results obtained through these two modifications are shown in Figures 10-13. The weighted IC consensus is achieved, which is shown in Figure $\mathbf{1 0}$ in the case of dynamic weights. The generations remain within the predefined ranges and updated for an optimal solution (Figure 11). The profiles for weights for the proposed modified approach are demonstrated in Figure 12, while the total demand remains unchanged, as per Figure 13. Hence, the use of weights can be more meaningful for a distributed EDP; however, at the same time, it can require more efforts and investigation to attain distributed EDP for complex systems, such as IEEE-118 bus system.

\section{CONCLUSION}

In this paper, a distributed EDP has been solved under bidirectional communication topology by incorporating weight adjustment. Two optimality conditions are provided: First condition encompasses a generic case and second condition encompasses distributed optimization. A distributed optimization protocol by using weighted ICs has been provided to deal with several constraints. A coupling weight selection approach for optimal solution of the weighted EDP has been derived via the sign-consensus method, supply-demand balance realization, proposed protocol analysis, generation dynamics investigation, and Lyapunov analysis. The proposed method has been applied to IEEE-30 bus and IEEE-118 bus systems, and it was observed that

\section{REFERENCES}

Ansari, A. H., Cherian, P. J., Caicedo, A., Naulaers, G., De Vos, M., and Van Huffel, S. (2019). Neonatal Seizure Detection Using Deep Convolutional Neural Networks. Int. J. Neur. Syst. 29, 1850011. doi:10.1142/s0129065718500119

Chansareewittaya, S. (2018). "Hybrid Ba/ats for Economic Dispatch Problem," in 2018 22nd International Computer Science and Engineering Conference (ICSEC), Chiang Mai, Thailand, 21-24 November 2018 (IEEE), 1-4. doi:10. 1109/icsec.2018.8712630

Chen, C., Bao, Y.-Q., Wu, X.-H., Wang, B., and Shen, C. (2019). Battery Energy Storage System Based on Incremental Cost Consensus Algorithm for the Frequency Control. IEEE Access 7, 147362-147372. doi:10.1109/access.2019. 2946458

Chen, C., Bao, Y.-Q., Wu, X.-H., and Wang, B. (2020). Incremental Cost Consensus Algorithm for On/off Loads to Enhance the Frequency Response of the Power System. IEEE Access 8, 67687-67697. doi:10.1109/access.2020.2985782

Chiarelli, A. M., Croce, P., Assenza, G., Merla, A., Granata, G., Giannantoni, N. M., et al. (2020). Electroencephalography-Derived Prognosis of Functional Recovery in Acute Stroke through Machine Learning Approaches. Int. J. Neural Syst. 30, 2050067. doi:10.1142/s0129065720500677

Dev, A., and Sarkar, M. K. (2019). Robust Higher Order Observer Based NonLinear Super Twisting Load Frequency Control for Multi Area Power Systems via Sliding Mode. Int. J. Control. Autom. Syst. 17, 1814-1825. doi:10.1007/ s12555-018-0529-4 the proposed method can be applied to attain an environmentally friendly solution of EDP. In addition, it can be used for the validation of capacity constraints when compared with the existing schemes. In the future, the proposed study can be extended to deal with the optimization of a more general objective function, containing the environmental effects.

\section{DATA AVAILABILITY STATEMENT}

The original contributions presented in the study are included in the article/Supplementary Materials, further inquiries can be directed to the corresponding authors.

\section{AUTHOR CONTRIBUTIONS}

WA, K-SH, and MR conceived of the idea. U-E-HA and WA developed the theoretical framework. U-E-HA, WA, K-SH, and MR verified the analytical methods. U-E-HA and SA performed the simulation results. U-E-HA, WA, and MR wrote the manuscript with support from K-SH and SA. U-E-HA, WA, and $\mathrm{K}-\mathrm{SH}$ checked the paper. All authors discussed the results and contributed to the final manuscript.

\section{FUNDING}

This work was supported by the Higher Education Commission (HEC) of Pakistan through indigenous PhD scholarship of the first author and by the National Research Foundation (NRF) of Korea under the auspices of the Ministry of Science and ICT, Korea (grant no. NRF-2020R1A2B5B03096000).

Dong, R., and Wang, S. (2020). New Optimization Algorithm Inspired by Kernel Tricks for the Economic Emission Dispatch Problem with Valve point. IEEE Access 8, 16584-16594. doi:10.1109/ACCESS.2020.2965725

El-Baz, W., Tzscheutschler, P., and Wagner, U. (2019). Evaluation of Energy Market Platforms Potential in Microgrids: Scenario Analysis Based on a Double-Sided Auction. Front. Energ. Res. 7, 41. doi:10.3389/fenrg.2019.00041

Flore, A., Clausen, M., and Uslar, M. (2019). Migration Paths for Smart Grids and Their Evaluation Using a Case Study. Front. Energ. Res. 7, 134. doi:10.3389/ fenrg.2019.00134

Gao, Y.-F., and Liu, L. (2020). Lyapunov-Based Triggering Mechanisms for EventTriggered Control. Int. J. Control. Autom. Syst. 18, 1392-1398. doi:10.1007/ s12555-019-0340-x

Gomez-Tames, J., Hirata, A., Tamura, M., and Muragaki, Y. (2019). Corticomotoneuronal Model for Intraoperative Neurophysiological Monitoring during Direct Brain Stimulation. Int. J. Neur. Syst. 29, 1850026. doi:10.1142/s0129065718500260

Guozden, T., Carbajal, J. P., Bianchi, E., and Solarte, A. (2020). Optimized Balance between Electricity Load and Wind-Solar Energy Production. Front. Energ. Res. 8, 16. doi:10.3389/fenrg.2020.00016

He, X., Yu, J., Huang, T., and Li, C. (2019). Distributed Power Management for Dynamic Economic Dispatch in the Multimicrogrids Environment. IEEE Trans. Contr. Syst. Technol. 27, 1651-1658. doi:10.1109/tcst.2018.2816902

Hou, H., Zhang, X., and Meng, Q. (2020). Olfactory Eeg Signal Classification Using a Trapezoid Difference-Based Electrode Sequence Hashing Approach. Int. J. Neur. Syst. 30, 2050011. doi:10.1142/s0129065720500112 
Hu, X., Zhang, H., Ma, D., and Wang, R. (2021). A Tngan-Based Leak Detection Method for Pipeline Network Considering Incomplete Sensor Data. IEEE Trans. Instrum. Meas. 70, 1-10. doi:10.1109/TIM.2020.3045843

IIT-Power-Group (2003). Ieee-118 Bus System Data by IIT Power Group, Electrical and Computer Engineering Department. Chicago: Illinois Institute of Technology.

Iqbal, M., Rehan, M., and Hong, K.-S. (2018). Robust Adaptive Synchronization of Ring Configured Uncertain Chaotic FitzHugh-Nagumo Neurons under Direction-Dependent Coupling. Front. Neurorobot. 12, 6. doi:10.3389/fnbot. 2018.00006

Jiang, Y., Zhang, H., and Chen, J. (2017). Sign-Consensus of Linear Multi-Agent Systems over Signed Directed Graphs. IEEE Trans. Ind. Electron. 64, 5075-5083. doi:10.1109/tie.2016.2642878

Krishnamurthy, S., Tzoneva, R., and Apostolov, A. (2017). Method for a Parallel Solution of a Combined Economic Emission Dispatch Problem. Electric Power Components Syst. 45, 393-409. doi:10.1080/15325008.2016.1265614

Lewis, F. L., Zhang, H., Hengster-Movric, K., and Das, A. (2014). Cooperative Control of Multi-Agent Systems: Optimal and Adaptive Design Approaches. London: Springer Publishing Company, Incorporated.

Liu, J., Chen, W., and Dai, H. (2016). Sampled-Data Based Distributed Convex Optimization with Event-Triggered Communication. Int. J. Control. Autom. Syst. 14, 1421-1429. doi:10.1007/s12555-015-0133-9

Liu, Y., Qu, Z., Xin, H., and Gan, D. (2017). Distributed Real-Time Optimal Power Flow Control in Smart Grid. IEEE Trans. Power Syst. 32, 3403-3414. doi:10. 1109/TPWRS.2016.2635683

Liu, G., Jiang, T., Ollis, T. B., Zhang, X., and Tomsovic, K. (2019). Distributed Energy Management for Community Microgrids Considering Network Operational Constraints and Building thermal Dynamics. Appl. Energ. 239, 83-95. doi:10.1016/j.apenergy.2019.01.210

Liu, Y., Li, Y., Xin, H., Gooi, H. B., and Pan, J. (2019). Distributed Optimal Tie-Line Power Flow Control for Multiple Interconnected Ac Microgrids. IEEE Trans. Power Syst. 34, 1869-1880. doi:10.1109/TPWRS.2018.2883407

Liu, X., Sun, C.-X., Gao, J., and Xu, S.-Y. (2020). Controllability of Networks of Multiple Coupled Neural Populations: An Analytical Method for Neuromodulation's Feasibility. Int. J. Neur. Syst. 30, 2050001. doi:10.1142/ s012906572050001x

Lü, Q., Liao, X., Li, H., and Huang, T. (2020). Achieving Acceleration for Distributed Economic Dispatch in Smart Grids over Directed Networks. IEEE Trans. Netw. Sci. Eng. 7, 1988-1999. doi:10.1109/tnse.2020.2965999

Ma, X., Yu, N., and Zhou, W. (2019). Using Dictionary Pair Learning for Seizure Detection. Int. J. Neur. Syst. 29, 1850005. doi:10.1142/s0129065718500053

Manzanera, O. M., Meles, S. K., Leenders, K. L., Renken, R. J., Pagani, M., Arnaldi, D., et al. (2019). Scaled Subprofile Modeling and Convolutional Neural Networks for the Identification of parkinsonâ $€^{\mathrm{TM}}$ s Disease in $3 \mathrm{~d}$ Nuclear Imaging Data. Int. J. Neural Syst. 29, 1950010. doi:10.1142/s0129065719500102

Mao, S., Dong, Z., Schultz, P., Tang, Y., Meng, K., Dong, Z. Y., et al. (2019). A Finite-Time Distributed Optimization Algorithm for Economic Dispatch in Smart Grids. IEEE Trans. Syst. Man, Cybernetics: Syst. 51 (4), 2068-2079. doi:10.1109/TSMC.2019.2931846

McLarty, D., Panossian, N., Jabbari, F., and Traverso, A. (2019). Dynamic Economic Dispatch Using Complementary Quadratic Programming. Energy 166, 755-764. doi:10.1016/j.energy.2018.10.087

Perng, J.-W., Kuo, Y.-C., and Lu, K.-C. (2020). Design of the Pid Controller for Hydro-Turbines Based on Optimization Algorithms. Int. J. Control. Autom. Syst. 18, 1758-1770. doi:10.1007/s12555-019-0254-7

Pintelon, R., Schoukens, J., and Vandersteen, G. (1997). Model Selection through a Statistical Analysis of the Global Minimum of a Weighted Nonlinear Least Squares Cost Function. IEEE Trans. Signal. Process. 45, 686-693. doi:10.1109/ 78.558486

Pourbabak, H., Alsafasfeh, Q., and Su, W. (2020). A Distributed Consensus-Based Algorithm for Optimal Power Flow in De Distribution Grids. IEEE Trans. Power Syst. 35, 3506-3515. doi:10.1109/TPWRS.2020.2974957

Pourbabak, H., Alsafasfeh, Q., and Su, W. (2019). Fully Distributed Ac Optimal Power Flow. IEEE Access 7, 97594-97603. doi:10.1109/ACCESS.2019.2930240

Rehan, M., Hong, K.-S., and Aqil, M. (2011). Synchronization of Multiple Chaotic FitzHugh-Nagumo Neurons with gap Junctions under External Electrical Stimulation. Neurocomputing 74, 3296-3304. doi:10.1016/j.neucom.2011.05.015
Rehan, M., and Hong, K.-S. (2011). LMI-Based Robust Adaptive Synchronization of FitzHugh-Nagumo Neurons with Unknown Parameters under Uncertain External Electrical Stimulation. Phys. Lett. A 375, 1666-1670. doi:10.1016/j. physleta.2011.03.012

Santos, C., Espinosa, F., Santiso, E., and Gualda, D. (2020). Lyapunov SelfTriggered Controller for Nonlinear Trajectory Tracking of Unicycle-type Robot. Int. J. Control. Autom. Syst. 18, 1829-1838. doi:10.1007/s12555-0180576-x

Schubert, R., and Stadelmann, M. (2015). Energy-Using Durables Â€" Why Consumers Refrain from Economically Optimal Choices. Front. Energ. Res. 3, 7. doi:10.3389/fenrg.2015.00007

Shadmand, M. B., Jain, S., and Balog, R. S. (2019). Autotuning Technique for the Cost Function Weight Factors in Model Predictive Control for Power Electronic Interfaces. IEEE J. Emerg. Sel. Top. Power Electron. 7, 1408-1420. doi:10.1109/JESTPE.2018.2849738

Shi, X., Zheng, R., Lin, Z., and Yan, G. (2020). Distributed Optimization for Economic Power Dispatch with Event-Triggered Communication. Asian J. Control. 22, 2412-2421. doi:10.1002/asjc.2140

Shi, X., Zheng, R., Lin, Z., Yang, T., and Yan, G. (2021). An Exponentially Convergent Distributed Algorithm for Resource Allocation Problem. Asian J. Control. 23, 1072-1082. doi:10.1002/asjc.2341

Souza, M. B. A., de Melo Honório, L., de Oliveira, E. J., and Moreira, A. P. G. M. (2020). Recursive Approach of Sub-Optimal Excitation Signal Generation and Optimal Parameter Estimation. Int. J. Control. Autom. Syst. 18, 1965-1974. doi:10.1007/s12555-019-0164-8

Sun, Q., Han, R., Zhang, H., Zhou, J., and Guerrero, J. M. (2015). A MultiagentBased Consensus Algorithm for Distributed Coordinated Control of Distributed Generators in the Energy Internet. IEEE Trans. Smart Grid 6, 3006-3019. doi:10.1109/TSG.2015.2412779

Tang, L., and Li, D. (2019). Time-varying Barrier Lyapunov Function Based Adaptive Neural Controller Design for Nonlinear Pure-Feedback Systems with Unknown Hysteresis. Int. J. Control. Autom. Syst. 17, 1642-1654. doi:10.1007/s12555-018-0745-y

Toh, K.-A., and Eng, H.-L. (2008). Between Classification-Error Approximation and Weighted Least-Squares Learning. IEEE Trans. Pattern Anal. Mach. Intell. 30, 658-669. doi:10.1109/TPAMI.2007.70730

us Saqib, N., Rehan, M., Iqbal, N., and Hong, K.-S. (2018). Static Antiwindup Design for Nonlinear Parameter Varying Systems with Application to Dc Motor Speed Control under Nonlinearities and Load Variations. IEEE Trans. Contr. Syst. Technol. 26, 1091-1098. doi:10.1109/TCST.2017.2692745

Wang, A., and Liu, W. (2020). Distributed Incremental Cost Consensus-Based Optimization Algorithms for Economic Dispatch in a Microgrid. IEEE Access 8, 12933-12941. doi:10.1109/access.2020.2966078

Wang, J., Li, H., and Wang, Z. (2019). Distributed Event-Triggered Scheme for Economic Dispatch in Power Systems with Uncoordinated Step-Sizes. IET Generation, Transm. Distribution 13, 3612-3622. doi:10.1049/iet-gtd.2019.0144

Wang, R., Sun, Q., Hu, W., Li, Y., Ma, D., and Wang, P. (2021). Soc-based Droop Coefficients Stability Region Analysis of the Battery for Stand-Alone Supply Systems with Constant Power Loads. IEEE Trans. Power Electron. 36, 7866-7879. doi:10.1109/TPEL.2021.3049241

Wang, Y., Wu, L., and Wang, S. (2017). A Fully-Decentralized Consensus-Based Admm Approach for Dc-Opf with Demand Response. IEEE Trans. Smart Grid 8, 2637-2647. doi:10.1109/TSG.2016.2532467

Wang, Z., Zheng, L., and Li, H. (2020). Distributed Optimization over General Directed Networks with Random Sleep Scheme. Int. J. Control. Autom. Syst. 18, 2534-2542. doi:10.1007/s12555-018-9543-9

Wei, Q., Zhu, S., Wang, Y., Gao, X., Guo, H., and Wu, X. (2020). A Training DataDriven Canonical Correlation Analysis Algorithm for Designing Spatial Filters to Enhance Performance of Ssvep-Based Bcis. Int. J. Neur. Syst. 30, 2050020. doi:10.1142/s0129065720500203

Wu, Q., Zhang, Y., Liu, J., Sun, J., Cichocki, A., and Gao, F. (2019). Regularized Group Sparse Discriminant Analysis for P300-Based Brain-Computer Interface. Int. J. Neur. Syst. 29, 1950002. doi:10.1142/ s0129065719500023

Wu, R., and Li, J. (1998). Time-Delay Estimation via Optimizing Highly Oscillatory Cost Functions. IEEE J. Oceanic Eng. 23, 235-244. doi:10. $1109 / 48.701196$ 
Xie, M., Gulzar, M. M., Tehreem, H., Javed, M. Y., and Rizvi, S. T. H. (2020). Automatic Voltage Regulation of Grid Connected Photovoltaic System Using Lyapunov Based Sliding Mode Controller: A Finite - Time Approach. Int. J. Control. Autom. Syst. 18, 1550-1560. doi:10.1007/ s12555-019-0563-x

Xing, H., Zeng, P., Mou, Y., and Wu, Q. (2019). Consensus-Based Distributed Approach to Lossy Economic Power Dispatch of Distributed Energy Resources. Int. Trans. Electr. Energ. Syst. 29, e12041. doi:10.1002/2050-7038.12041

Yang, D., Hong, K.-S., Yoo, S.-H., and Kim, C.-S. (2019). Evaluation of Neural Degeneration Biomarkers in the Prefrontal Cortex for Early Identification of Patients with Mild Cognitive Impairment: An Fnirs Study. Front. Hum. Neurosci. 13, 317. doi:10.3389/fnhum.2019.00317

Yang, D., Huang, R., Yoo, S.-H., Shin, M.-J., Yoon, J. A., Shin, Y.-I., et al. (2020). Detection of Mild Cognitive Impairment Using Convolutional Neural Network: Temporal-Feature Maps of Functional Near-Infrared Spectroscopy. Front. Aging Neurosci. 12, 141. doi:10.3389/fnagi.2020.00141

Yang, L., Luo, J., Xu, Y., Zhang, Z., and Dong, Z. (2020). A Distributed Dual Consensus Admm Based on Partition for Dc-Dopf with Carbon Emission Trading. IEEE Trans. Ind. Inf. 16, 1858-1872. doi:10.1109/TII.2019. 2937513

Yao, L., Wang, Z., Wang, Q., Xia, J., and Shen, H. (2020). Exponential Stabilization of Delayed Complex-Valued Neural Networks with Aperiodic Sampling: A Free-Matrix-Based Time-Dependent Lyapunov Functional Method. Int. J. Control. Autom. Syst. 18, 1894-1903. doi:10.1007/s12555019-0706-0

Yi, Z., Xu, Y., Hu, J., Chow, M.-Y., and Sun, H. (2020). Distributed, NeurodynamicBased Approach for Economic Dispatch in an Integrated Energy System. IEEE Trans. Ind. Inf. 16, 2245-2257. doi:10.1109/tii.2019.2905156

Yin, Z., Jiang, X., and Wang, F. (2020). Stability Criteria for Systems with Multiple Probabilistic Intervals Time-Varying Delay. Int. J. Control. Autom. Syst. 18, 877-885. doi:10.1007/s12555-019-0309-9

Yu, M., Song, C., Feng, S., and Tan, W. (2020). A Consensus Approach for Economic Dispatch Problem in a Microgrid with Random Delay Effects. Int. J. Electr. Power Energ. Syst. 118, 105794. doi:10.1016/j.ijepes.2019. 105794

Yu, W., Li, C., Yu, X., Wen, G., and Lü, J. (2015). “Distributed Consensus Strategy for Economic Power Dispatch in a Smart Grid,” in 2015 10th Asian Control Conference (ASCC), 1-6. doi:10.1109/ASCC.2015.7244550

Yun, H., Shim, H., and Ahn, H.-S. (2019). Initialization-Free Privacy-Guaranteed Distributed Algorithm for Economic Dispatch Problem. Automatica 102, 86-93. doi:10.1016/j.automatica.2018.12.033
Zafar, A., and Hong, K.-S. (2020). Reduction of Onset Delay in Functional NearInfrared Spectroscopy: Prediction of Hbo/hbr Signals. Front. Neurorobot. 14, 10. doi:10.3389/fnbot.2020.00010

Zhang, Q., Gong, Z., Yang, Z., and Chen, Z. (2019). Distributed Convex Optimization for Flocking of Nonlinear Multi-Agent Systems. Int. J. Control. Autom. Syst. 17, 1177-1183. doi:10.1007/s12555-018-0191-x

Zhang, X. S., Li, Q., Yu, T., and Yang, B. (2018). Consensus Transfer Q -Learning for Decentralized Generation Command Dispatch Based on Virtual Generation Tribe. IEEE Trans. Smart Grid 9, 2152-2165. doi:10.1109/TSG.2016.2607801

Zhang, X., Xu, H., Yu, T., Yang, B., and Xu, M. (2016). Robust Collaborative Consensus Algorithm for Decentralized Economic Dispatch with a Practical Communication Network. Electric Power Syst. Res. 140, 597-610. doi:10.1016/j. epsr.2016.05.014

Zhao, Y., Zhang, Y., and Lee, J. (2019). Lyapunov and Sliding Mode Based LeaderFollower Formation Control for Multiple mobile Robots with an Augmented Distance-Angle Strategy. Int. J. Control. Autom. Syst. 17, 1314-1321. doi:10. 1007/s12555-018-0194-7

Zhao, Z., Chen, G., and Dai, M. (2018). Distributed Event-Triggered Scheme for a Convex Optimization Problem in Multi-Agent Systems. Neurocomputing 284, 90-98. doi:10.1016/j.neucom.2017.12.060

Zhu, M., Yang, Q., Dong, J., Zhang, G., Gou, X., Rong, H., et al. (2021). An Adaptive Optimization Spiking Neural P System for Binary Problems. Int. J. Neural Syst. 31, 2050054. doi:10.1142/s0129065720500549

Conflict of Interest: The authors declare that the research was conducted in the absence of any commercial or financial relationships that could be construed as a potential conflict of interest.

Publisher's Note: All claims expressed in this article are solely those of the authors and do not necessarily represent those of their affiliated organizations, or those of the publisher, the editors and the reviewers. Any product that may be evaluated in this article, or claim that may be made by its manufacturer, is not guaranteed or endorsed by the publisher.

Copyright (c) 2022 Alvi, Ahmed, Hong, Rehan and Ahmed. This is an open-access article distributed under the terms of the Creative Commons Attribution License (CC $B Y)$. The use, distribution or reproduction in other forums is permitted, provided the original author(s) and the copyright owner(s) are credited and that the original publication in this journal is cited, in accordance with accepted academic practice. No use, distribution or reproduction is permitted which does not comply with these terms. 